Military Technical College Kobry El-Kobba

Cairo, Egypt

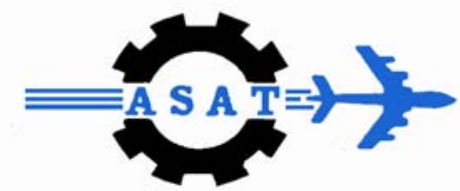

12-th International Conference on Aerospace Sciences \& Aviation Technology

\title{
INVERSE APPROACH SOLUTION FOR TURBULENT BOUNDARY LAYERS SUBJECTED TO ARBITRARY PRESSURE GRADIENTS
}

\author{
Mohammed Elhadi Ahmed Elsayed!
}

\begin{abstract}
This paper deals with the study of the turbulent boundary layer development on two dimensional flows subjected to steady incompressible oncoming stream. In the turbulent flow, the boundary layer is divided into two layers, a wall layer and an outer layer. It is well known that the wall layer is universal but the outer layer depends on the pressure gradient. The outer layer is modeled as a nonlinear turbulent wake and consequently a constant eddy viscosity model for Clauser is adopted. The outer layer problem is shown to be governed by the classical Falkner - Skan equation for the laminar wake, the difference being in a turbulent condition which requires a finite stress at its inner boundary instead of zero stress in laminar flows. For the computations it was convenient to convert the above direct problem (for a given stress, find the slip velocity at the wall) to an inverse problem (given the slip velocity at the wall find the stress). Riley \& Weidman (1989) dealt with the solution of the above inverse problem, but had not however covered the entire range of solutions. In the present work a much wider range solution to the inverse problem using a finite domain transformation is obtained by Runge-Kutta method. The results are displayed graphically and discussed critically. The solution of the inner and outer layers put together describes the turbulent boundary layer subjected to arbitrary pressure gradients.
\end{abstract}

\section{KEY WORDS:}

Turbulent boundary layer, eddy viscosity model, finite stress, slip velocity, inverse problem, pressure gradients.

! Assist. Professor, Karary Academy of Technology -Khartoum - Sudan, P.O. Box: 12304 Khartoum, Tel.: (249)-122233908, Fax: (249)-87-239000, Email: mohadi20@hotmail.com 


\section{INTRODUCTION}

Turbulence can be defined as a chaotic vorticity field with the statistical mean being deterministic. Osborne Reynold was the first to recognize that turbulence is a problem in statistical dynamics. He separated the mean and fluctuating quantities by defining a probability average such that the mean stream is interacting with the fluctuating irregular edding indeterministic motion. His approach has lead to Reynolds's equations which involve additional unknowns called Reynold's stresses.

Much of what is known about turbulent shear flows stems from experiments supplemented by dimensional and similarity arguments.

\section{D Turbulent boundary layer equations}

The turbulent boundary layer equations for a steady homogeneous isotropic Newtonian incompressible two dimensional flows in rectangular Cartesian coordinates are

\section{Continuity equations}

$$
\partial u / \partial x+\partial v / \partial y=0
$$

\section{Momentum equation}

$$
u \partial u / \partial x+v \partial u / \partial y-U_{e} d U_{e} / d x=d \tau / d y+v \partial^{2} u / \partial y^{2}
$$

The boundary conditions are

$$
\begin{array}{ll}
y=0, & u=v=\tau=0 \\
y \rightarrow \infty, & u \rightarrow U_{e}(x), \quad \tau \rightarrow 0
\end{array}
$$

The analysis of turbulent boundary layer equations in the two layers is described below [2];

\section{Inner layer}

Introducing these inner variables

$$
\begin{aligned}
& u=u_{\tau} f\left(y_{+}\right) \\
& \tau=\tau_{w} g\left(y_{+}\right) \\
& y_{+}=y u / v
\end{aligned}
$$

into the boundary layer equations (1) and (2) the lowest order terms give after integration;

$$
\partial^{2} f / \partial y_{+}^{2}+g=1
$$


i.e., the total stress in the inner layer is constant.

\section{Outer Layer}

The outer variables are

$$
\begin{aligned}
& Y=y / \delta \\
& u=U_{e}(X) \partial F(X, Y) / \partial Y \\
& \tau_{w}=\rho u_{\tau}^{2} T(X, Y) \\
& \delta / L=\left(u_{\tau} / U_{e}\right)^{2} \\
& X=\int_{0}^{x} u_{\tau}^{2} d X / \delta U_{e}^{2}
\end{aligned}
$$

In the outer variables the boundary layer equations reduce to

$\frac{U_{e x}}{U_{e}} F^{\prime 2}-\frac{+\delta_{X}}{\delta} F F^{\prime \prime}+F F_{X}^{\prime}-F_{X} F^{\prime \prime}-\frac{U_{e X}}{U_{e}} F F^{\prime \prime}=\frac{U_{e X}}{U_{e}}+\frac{\partial T}{\partial Y}+v \frac{U_{e}}{\delta^{2} u_{\tau}^{2}} F^{\prime \prime \prime}$

subjected to the boundary conditions

$$
Y \rightarrow \infty, F^{\prime} \rightarrow 1, T \rightarrow 0
$$

The expansions in the outer layer are

$$
\begin{aligned}
& F=F_{o}(X, Y)+\gamma F_{1}(X, Y)+\ldots . . \\
& T=T_{o}(X, Y)+\gamma T(X, Y)+\ldots . .
\end{aligned}
$$

where $\gamma$ is a gauge function. The equation satisfied by the lowest order terms is

$$
\frac{U_{e x}}{U_{e}} F_{o}^{\prime 2}-\frac{\delta_{x}}{\delta} F_{o} F_{o}^{\prime \prime}+F_{o} F_{o x}^{\prime}-F_{o x} F_{o}^{\prime \prime}-\frac{U_{e X}}{U_{e}} F_{o} F_{o}^{\prime \prime}=\frac{U_{e X}}{U_{e}}+\frac{\partial T_{o}}{\partial Y}
$$

subjected to the boundary conditions

$$
\begin{aligned}
& Y \rightarrow 0, \quad F_{o} \rightarrow 0, T_{o} \rightarrow 1 \\
& Y \rightarrow \infty, \quad F_{o}^{\prime} \rightarrow 1, T_{o} \rightarrow 0
\end{aligned}
$$

\section{The Matching}

The matching for the tangential velocity component leads to the functional equation [3];

$u_{\tau} f\left(y_{+} \rightarrow \infty\right) \approx \partial F_{o}(X, Y \rightarrow 0) / \partial Y+\gamma \partial F_{1}(X, Y \rightarrow 0) / \partial Y$ 
$\partial F_{o}(X, 0) / \partial Y=G_{o}(X)$ is the slip velocity due to the outer inviscid solution at the wall, expected of order unity except for very strong pressure gradients. The term $f\left(y_{+}\right)$must diverge; say as $g\left(y_{+}\right)$at large $y$ to balance the slip velocity term. Also the term $\partial F_{1} / \partial Y$ should diverge as $Y \rightarrow 0$, say as $G_{1}=(X, Y)$.

Equation (12) then becomes;

$u_{\tau} g\left(y_{+}\right) \approx G(X)+\gamma_{o} G_{1}(X, Y)$

Differentiation w.r.t. $Y$ gives

$u_{\tau} y_{+} \partial g / \partial y_{+} \approx Y \partial G_{o} / \partial Y+\gamma_{o} Y \partial G_{1} / \partial Y$

The matching to the lowest order gives

$Y \partial G_{o} / \partial Y=0 \quad$ at $Y=0$

Assuming $\gamma=u_{\tau}$ the next order terms are

$y_{+} \partial g / \partial y_{+} \approx Y \partial G_{1} / \partial Y$

This shows that both sides approaches a constant, say like $1 / k$ independent of $y_{+}$and $Y$. From

$g=f\left(y_{+} \rightarrow \infty\right)$
$G=\partial F_{1}(X, Y \rightarrow 0) / \partial Y$

we get

$$
\begin{aligned}
& f\left(y_{+}\right)=u / u_{\tau}=(1 / k) \ln y_{+}+A \\
& \partial F_{1} / \partial Y=(1 / k) \ln Y-B
\end{aligned}
$$

The matching of the tangential velocity gives

$$
F_{o}^{\prime}(X, 0) / u_{\tau}=(1 / k) \ln \delta_{+}+A+B
$$

which is the skin friction law. Subtracting (20) from (18) we get

$$
\left(F_{o}^{\prime}(X, 0)-u\right) / u_{\tau}=-(1 / k) \ln Y+B
$$

From

$U_{1}(X, 0)=F_{o}^{\prime}(X, 0)$ 
we write

$U_{1}(X, 0) / u_{\tau}=(1 / k) \ln \delta_{+}+A+B$

Equation (18) is the well known law of wall. Equation (21) is more general than the classical defect law and may be called the local defect law. To proceed further in the solutions of inner and outer layers we need some kind of Clauser hypothesis or certain evidences from experiments.

\section{Method of solution -Inner layer}

It is known from experiments that the log law is observed and substantial log region exists. Throughout this work a fix set of boundary layer constants $(k=0.41$ and $A=5)$ has been used. Therefore, the law of the wall in the inner layer, Equation (18), is

$$
u / u_{\tau}=(1 / .41) \ln y_{+}+5 \quad \text { for large } y
$$

For small $y$ the analysis of boundary layer equations shows that the velocity profile is linear such that;

$u / u_{\tau}=y_{+}$

The domain of change of the region from Equation (25) to Equation (24) is not known in closed form. But if we use an expression due to Spalding [4] then the velocity distribution in the entire inner layer can be assumed known. This relation due to Spalding is an empirical implicit correlation with the following form;

$$
y_{+}=1 / k+e^{-k c}\left(e^{\varsigma}-1-\varsigma-1 / 2 \varsigma^{2}-1 / 6 \varsigma^{3}-1 / 24 \varsigma^{4}\right), \quad \varsigma=k U_{e} / u_{\tau}
$$

\section{Method of solution-Outer layer}

The equation in the outer layer is

$$
\frac{U_{e X}}{U_{e}} F_{o}^{\prime 2}+F_{o X}^{\prime} F^{\prime}-F_{o X} F_{o}^{\prime \prime}-\left(\frac{\delta_{x}}{\delta}+\frac{U_{e X}}{U_{e}}\right) F_{o} F_{o}^{\prime \prime}=\frac{U_{e X}}{U_{e}}+\frac{\partial T_{o}}{\partial Y}
$$

With the boundary conditions at infinity

$$
Y \rightarrow \infty, F_{o}^{\prime} \rightarrow 1, T_{o} \rightarrow 0
$$

and the matching conditions with the inner layer

$$
Y=0, \quad F_{o}=0, \quad Y F_{o}^{\prime \prime} \rightarrow 0, \quad T_{o} \rightarrow 1
$$

In order to solve the outer layer we assume an eddy viscosity hypothesis 
$\tau=\rho v_{\tau} \partial u / \partial y$

Clauser [5] has assumed that the eddy Reynold's number $U_{e} \delta^{*} / v_{\tau}=R_{s}$, remains constant in the outer layer. Thus

$v_{\tau}=R_{s} \int_{0}^{\infty}\left(U_{e}-u\right) d y, \quad R_{s} \approx 50$

Equation (30) can also be written as

$T_{o}=\left(v_{\tau} U_{e} / \delta u_{\tau}^{2}\right) F_{o}^{\prime \prime}$

Therefore Equation (27) becomes

$\frac{U_{e x}}{U_{e}}\left(-1+F_{o}^{\prime 2}\right)-\left(\frac{\delta_{x}}{\delta}+\frac{U_{e x}}{U_{e}}\right) F_{o} F_{o}^{\prime \prime}+F_{o} F_{o x}^{\prime}-F_{o X} F_{o}^{\prime \prime}=\frac{v_{\tau}}{\delta^{2} U_{e}} F_{o}^{\prime \prime \prime}$

With the boundary conditions given as;

$Y=0, \quad F_{o}=0, F_{o}^{\prime}=1, \quad F_{o}^{\prime \prime}=\delta u_{\tau}^{2} / v_{\tau} U_{e}$

$Y \rightarrow \infty, F_{o}^{\prime} \rightarrow 1$

Let

$F_{o}(X, Y)=\alpha g(x, \varsigma), \quad Y=\alpha \varsigma$

Equation (33) can be written as

$\left(-1+g^{\prime 2}\right) \frac{U_{e x} / U_{e}}{\frac{\delta_{x}}{\delta}+\frac{U_{e x}}{U_{e}}}-g g^{\prime \prime}+\frac{1}{\frac{\delta_{x}}{\delta}+\frac{U_{e x}}{U_{e}}}\left(g g_{x}-g_{x} g^{\prime \prime}\right)=\frac{v_{\tau}}{\delta^{2} U_{e} \alpha^{2}\left(\frac{\delta_{x}}{\delta}+\frac{U_{e x}}{U_{e}}\right)} g^{\prime \prime \prime}$

If

$\beta=\frac{U_{e x} / U_{e}}{\frac{\delta_{x}}{\delta}+\frac{U_{e x}}{U_{e}}}, \quad \alpha^{2}=\frac{v_{\tau}}{\delta^{2} U_{e x}} \beta$

then Equation (36) is

$g^{\prime \prime \prime}+\beta\left(1-g^{\prime 2}\right)+g g^{\prime \prime}+\beta \frac{U_{e}}{U_{e x}}\left(g g_{x}-g_{x} g^{\prime \prime}\right)=0$ 
subjected to the boundary conditions

$$
\begin{aligned}
& \varsigma=0, \quad g=0, g_{o}^{\prime \prime}=\delta u_{\tau}^{2} \alpha / \nu_{\tau} U_{e} \\
& \varsigma \rightarrow \infty, g \rightarrow 1
\end{aligned}
$$

For equilibrium pressure gradients $\beta$ is constant and $\partial / \partial x=0$. Equation (38) becomes

$$
g^{\prime \prime \prime}+g g^{\prime \prime}+\beta\left(1-g^{\prime 2}\right)=0
$$

with the boundary conditions

$$
\begin{aligned}
& \varsigma=0, \quad g=0 \\
& g^{\prime \prime}(0)=C_{f} / 2 R_{s} \int_{0}^{\infty}(1-g) d \varsigma \\
& \varsigma \rightarrow \infty, \quad g \rightarrow 1
\end{aligned}
$$

Here $C_{f}$ is the skin friction and is given by

$$
C_{f}=2 R_{s} g^{\prime \prime}(0) \int_{0}^{\infty}(1-g) d \varsigma
$$

The condition $\beta$ is constant implies that

$$
U_{e} \alpha \delta^{a}
$$

where $a$ is a constant equals $\beta / 1-\beta$. This in turn implies that similar solutions of the boundary layer equations are obtained when the velocity distribution of the potential flow is proportional to a power of the boundary layer thickness. Clauser [5] in his classical work on the outer layer of the turbulent boundary layer has also obtained Equation (40). He prescribed the boundary conditions (41a) and (41c) and determined a family of the profiles of $f^{\prime}(0)$ when $\beta$ was held fixed. By actual joining of the experimental velocity profiles for the inner layer, he estimated the skin friction $C_{f}$.

The advantages of the present procedure are that we do not compute the family of the profiles and fix this arbitrariness by prescribing the boundary condition (41b). Equation (40) is the well known Falkner Skan laminar boundary layer equation. A finite stress arises from the fact that the outer layer has to match with the inner layer and as a consequent of matching a finite stress is imposed on the outer layer.

The equations (40) \& (41) form a two point boundary value problem governed by a third order ordinary differential equation. For a given pressure gradient $\beta$ and skin 
friction $C_{f}$ the equation can be numerically obtained to get the slip velocity $g^{\prime}(0)$. This is called the direct problem. In the turbulent flows, however, the slip velocity $g^{\prime}(0)$ is prescribed by the inner layer and therefore $C_{f}$ is to be determined from the solution. This problem is referred here as the inverse problem which can be stated as

$$
\begin{gathered}
g^{\prime \prime \prime}+g g^{\prime \prime}+\beta\left(1-g^{\prime 2}\right)=0 \\
g(0)=0, \quad g^{\prime}(0)=c, \quad g^{\prime}(\infty)=1
\end{gathered}
$$

For a given $\beta$ and $c$, the equation can be integrated to obtain the profile and $g^{\prime \prime}(0)$; consequently skin friction $C_{f}$ can be estimated from the Equation (42).

However, Riley \& Weidman [6], in their work on stretched boundaries, reported solutions for the ranges $-1 \leq \beta \leq+1$ and $-1 \leq c \leq+1$. Their solutions correspond to the case when the free stream is dominant compared with the stretching of the wall $(c \approx 1)$. Riley \& Weidman had not covered the situation when stretching is dominant compared with the free stream $(c>1$ or $c>>1)$, i.e. the magnitude of the free stream velocity becomes smaller than the stretching velocity at the wall. However, the situation of $c>>1$ would lead to difficulties in the numerical solution, which was resolved in this work by a finite transformation

$$
\begin{aligned}
& g(\varsigma)=f(\eta) / \sqrt{\varepsilon} \\
& \varsigma=\eta \sqrt{\varepsilon} \\
& \varepsilon=1 / 1+c=U_{\infty} /\left(U_{w}+U_{\infty}\right)
\end{aligned}
$$

Substituting the finite transformations, the inverse problem takes the form

$$
\begin{aligned}
& f^{\prime \prime \prime}+f f^{\prime \prime}+\beta\left(\varepsilon^{2}-f^{\prime 2}\right)=0 \\
& f(0)=0, \quad f^{\prime}(0)=1-\varepsilon, \quad f^{\prime}(\infty)=\varepsilon
\end{aligned}
$$

In terms of present variables $\beta$ and $\varepsilon$, the solution presented by Riley \& Weidman corresponds to $\beta \leq 1$ and $\varepsilon \geq 0.5$.

In the present work the solutions are obtained for $\beta \leq 2$ and $0 \leq \varepsilon \leq 1$. The equations (45) and (46) are integrated numerically by Runge Kutta method with a step size $\partial \eta=0.05$. The boundary conditions at infinity are satisfied by a shooting method which interpolates the two computed solutions so that the error in the boundary conditions at infinity becomes minimum in the least squares sense.

\section{Results \& Discussion}

Velocity profiles for various values $\varepsilon$ and $\beta$ are displayed on figures (1), (2) and (3) and for values of $\beta=1.0,0.5,0$ respectively. It is observed that as pressure gradient increases the velocity profiles converge quickly to the outer value $1-\varepsilon$. This means that boundary layer thickness decreases as pressure gradient increases. Fig. 1 
shows that for very large values of slip velocity $(\varepsilon=0)$, the non-dimensional tangential velocity component $f^{\prime}(\eta)$ starts from unity and decreases to zero asymptotically far enough from the wall where it matches smoothly with the outer value $\varepsilon$. Fig. 1 shows that in the region $0 \leq \varepsilon \leq 0.5, f^{\prime}(\eta)$ reaches its maximum at the wall and then decreases to the outer value. For various values of $\varepsilon$, at a certain distance from the wall, the velocity profiles fall together. This means that the tangential velocities are nearly the same regardless the slip velocity value.

Stream function distributions, $f(\eta)$ across the layer for various values of $\beta$ are shown in figures (1), (2) and (3). Fig. 1 shows that for $\varepsilon=0.4,0.6,0.8,1.0$, distribution of $f(\eta)$ is almost linear while for $\varepsilon=0$ it increases from zero at the wall to a certain value and then becomes constant through the remaining part of the layer. All figures are qualitatively same and so no additional commences are required except that for $\beta=0$, stream function near the wall is higher for $\varepsilon=0$ with the lowest being for $\varepsilon=1.0$.

Displacement thickness against $\varepsilon$ is displayed on Fig. 4 for positive $\beta$ and on Fig. 5 for negative $\beta$. It is observed that as $\beta$ increases, displacement thickness decreases, and that as the slip velocity decreases, displacement thickness increases. Displacement thickness is zero for $(\varepsilon=0.5)$, negative for $\varepsilon<0.5$ and positive for $\varepsilon>0.5$. Fig. 5 shows that at and beyond separation, displacement thickness increases faster and turns up at some value of $\varepsilon$.

Skin friction against $\varepsilon$ is displayed on Fig. 6 for positive pressure gradients $(\beta>0)$ and on Fig. 7 for negative pressure gradients $(\beta<0)$. In these figures, it is observed that skin friction is zero when the wall slip velocity is same as the free stream velocity ( $\varepsilon=0.5$ ), negative for $\varepsilon<0.5$ and positive for $\varepsilon>0.5$. For a fixed $\beta$, skin friction increases as wall slip velocity decreases. It is also observed that as the pressure gradient increases, skin friction increases. It is clear that flows with high pressure gradients and small or zero slip velocity values, will suffer high skin friction. Fig. 7 shows that at separation $(\beta=-0.19883)$, skin friction is maximum at about $\varepsilon=0.82$ and then decreases to zero at $\varepsilon=1.0$.

\section{Conclusions}

The present paper dealt with the analysis of two dimensional turbulent boundary layers with moderately high pressure gradients. For the computation, an inverse approach has been followed. The inverse problem can be stated as; given the slip velocity at the wall find the stress.

The present work succeeded to obtain solution for a wider range of pressure gradients and slip velocities using a finite domain transformation. The results showed that skin friction is zero when wall slip velocity is same as the free stream velocity, negative for $\varepsilon<0.5$ and positive for $\varepsilon>0.5$. For a fixed pressure gradient value, skin friction increases as wall slip velocity decreases. Flows with high pressure gradients 
and small or zero slip velocity values, will suffer high skin friction. At and beyond separation, displacement thickness increases faster and turns up at some value of $\varepsilon$. However, more work is required to assess and validate the above results. This is being conducted by the author presently.

However, the present work is still in the beginning. Validation of results needs to be conducted. Also, experimental work seems to be required, at least to measure the pressure distribution over a real surface - e.g., an airfoil surface-, from which the external velocity distribution could be evaluated, and substituted for to obtain the pressure gradient parameter. The interesting issue of the present work is that it deals with the solution of the problem of incompressible turbulent boundary layer with pressure gradients which is vital in the skin friction drag calculations for an airplane wings. These issues will be addressed later by the author.

\section{REFERENCES}

1. Schlichting. H., "Boundary Layer Theory", Mc Graw Hill, N.Y., (1978).

2. Townsend. A. A., "Structures of Turbulent Flows", Cambridge University Press, (1975).

3. Van Dyke, "Perturbation Methods in Fluid Mechanics", Academic Press, N.Y., (1975).

4. Spalding, D. B., "A Single Formula for the Law of the Wall", J. Applied Mechanics, Transaction of the ASME, Series E., Vol. 83, pp 455, (1961).

5. Clauser. F. H., "The Turbulent Boundary Layers. Advances in Applied Mechanics", Vol. 4, Academic Press, N.Y., (1956).

6. Riley. N and Weidman P. D., "Multiple Solutions of the Falkner Skan Equation for Flow Past a Stretching Boundary", SIAM J. Of Applied Math., Vol.49, pp 135, (1989). 


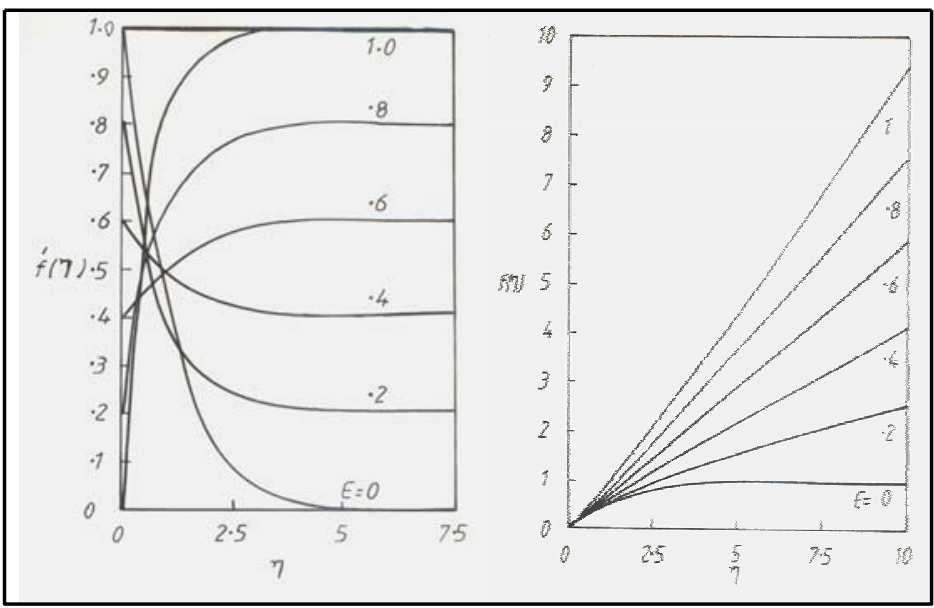

Fig. 1: Velocity $f^{\prime}(\eta)$ \& Stream Function $f(\eta)$ Distributions for $\beta=1$

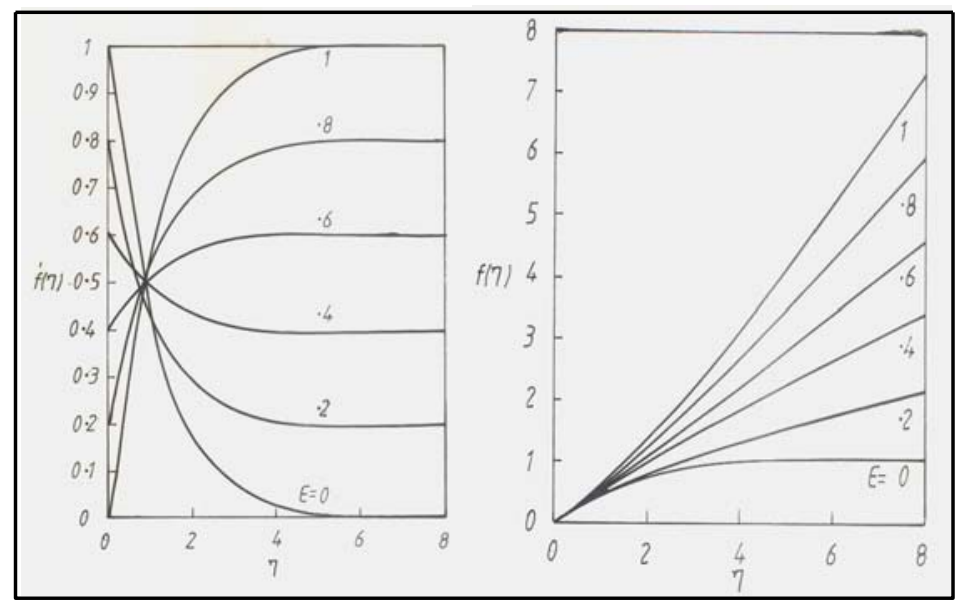

Fig. 2: Velocity $f^{\prime}(\eta)$ \& Stream Function $f(\eta)$ Distributions for $\beta=0.5$

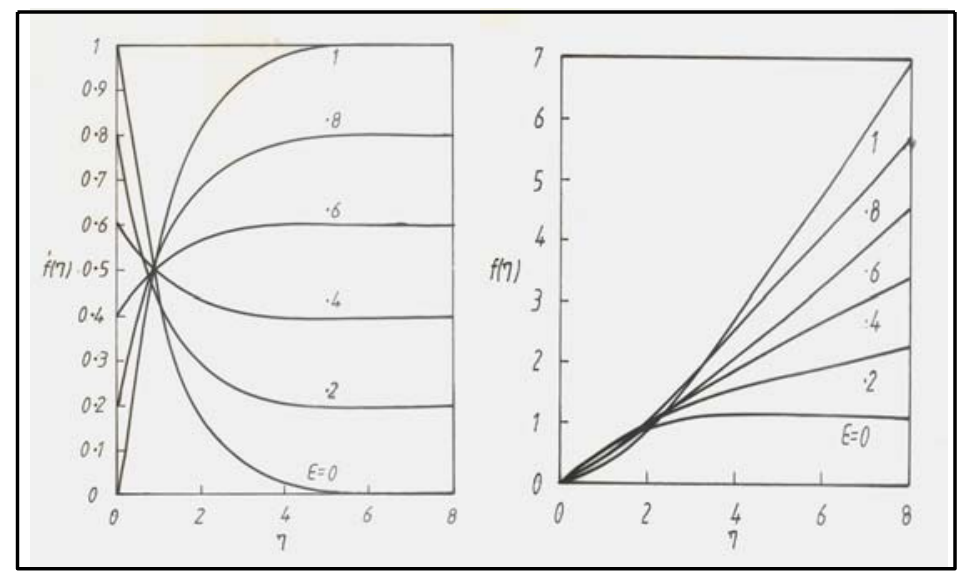

Fig. 3: Velocity $f^{\prime}(\eta)$ \& stream Function $f(\eta)$ Distributions for $\beta=0$ 


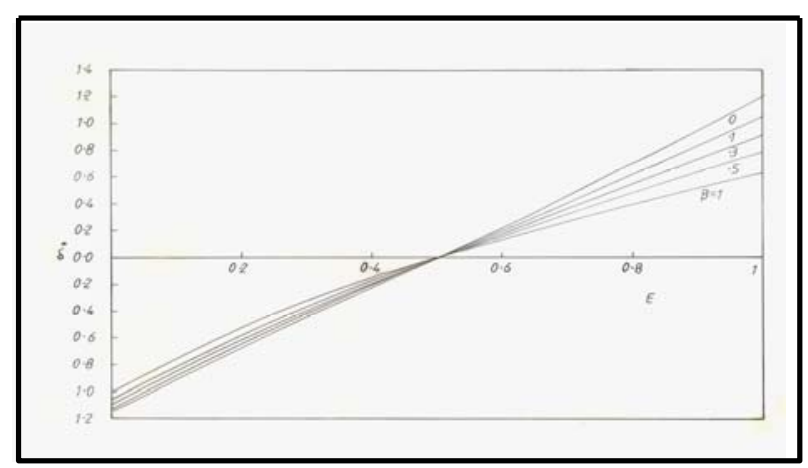

Fig. 4: Displacement thickness $\delta^{*}$ distribution against $\varepsilon$ for positive $\beta$

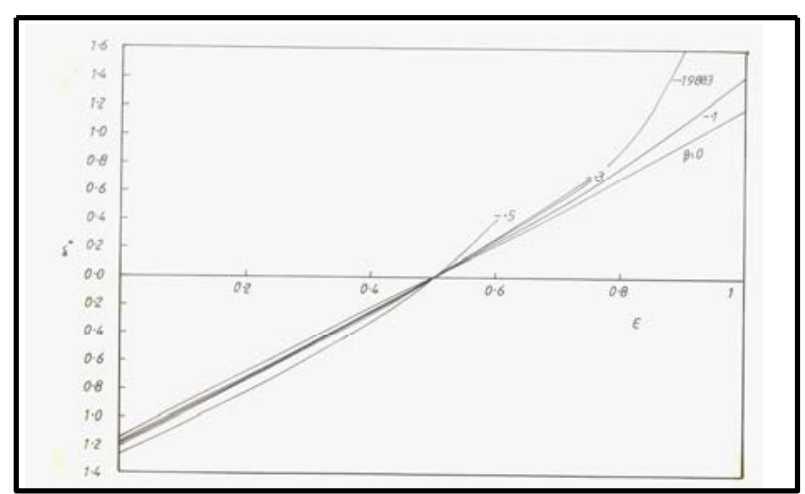

Fig. 5: Displacement thickness $\delta^{*}$ distribution against $\varepsilon$ for negative $\beta$

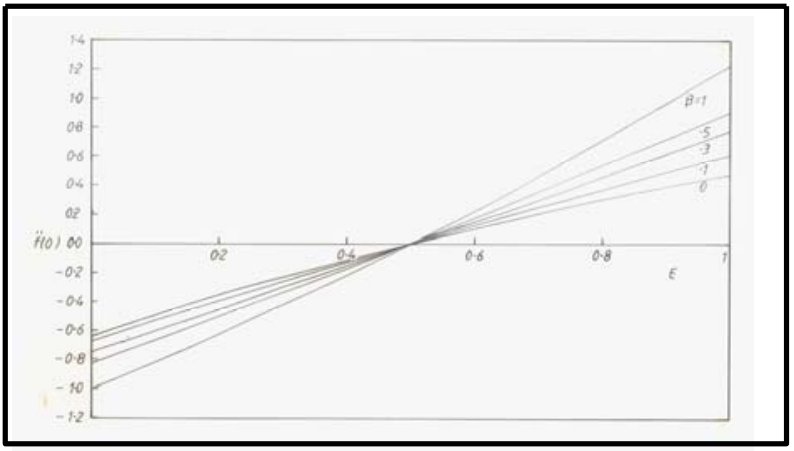

Fig. 6: Skin friction $f^{\prime \prime}(0)$ distribution against $\varepsilon$ for positive $\beta$

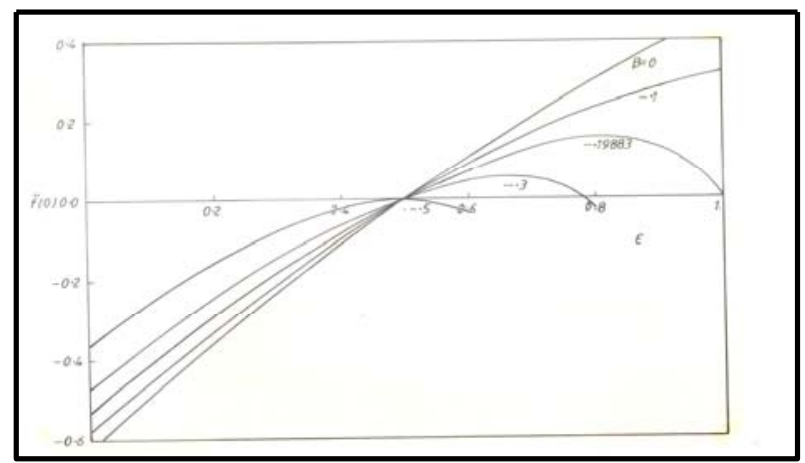

Fig. 7: Skin friction $f^{\prime \prime}(0)$ distribution against $\varepsilon$ for negative $\beta$ 